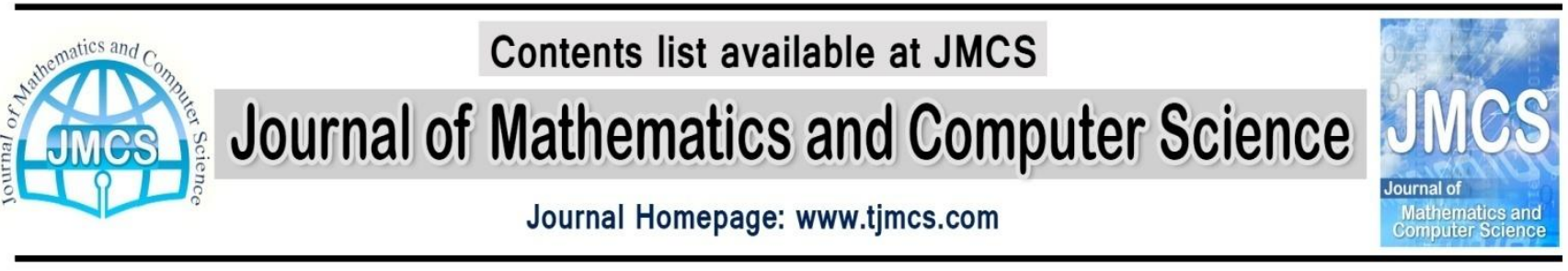

\title{
WKB and Numerical Compound Matrix Methods for Solving the Problem of Everted Neo-Hookean Spherical Shell Morteza Sanjaranipour ${ }^{1}$, Hamed Komeyli $i^{2}$
}

${ }^{1}$ Faculty Member of Sistan \& Balouchestan University, Zahedan, Iran

${ }^{2}$ MA of Applied Mathematics, Zahedan, Iran

mspour@hamoon.usb.ac.ir

Article history: hd.komeyli@yahoo.com

Received March 2014

Accepted April 2014

Available online June 2014

\section{Abstract}

The present paper deals with an eigenvalue problem which describes an everted neo-hookean spherical shell which its outer surface is deformed in compression under hydrostatic pressure. Our approach is based on mathematical modeling using a differential equation of order four and boundary conditions including two differential equations of order two and three. We solve the above mentioned problem using two different expansions of WKB method. We also investigate how to apply the numerical compound matrix on the problem and show the application of Runge-Kutta-Fehlberg and Newton-Raphson numerical algorithm. Finally, by comparing the data obtained from these two methods (numerical and WKB), we not only learn about the turning point, we also find out that the reason of the difference between the results of the two methods is this turning point.

Keywords: We compound matrix method, elasticity, incompressible, spherical, WKB method

\section{Introduction}

In 1999 Chen and Haughton developed a mathematical model for the everted spherical shell and using WKB method solved it for Varga elastic material. In 2003, they used the numerical compound method to solve the problem for Varga elastic material. In this paper, we try to solve the problem using the two expansions $S(r)=\sum_{i=0}^{2} \frac{1}{n^{i}} S_{i}$ and $R(r)=\sum_{i=0}^{2} \frac{1}{n^{i}} R_{i}$ of WKB method and obtained the only non-zero equilibrium condition of the problem. We also analyze the problem using the numerical compound method and by defining the compound variables, change a boundary value problem of order four to an

The corresponding author : H. Komeyli 
initial value problem with initial condition. Using numerical methods such as Runge-Kutta-Fehlberg and bisection method in Fortran 90, we solve this problem. Since we apply the numerical compound method for an eversion problem, the Fortran would be somehow different from other programs used for solving compound problems. After finding the eigenvalue of the problem, we use the numerical compound method again to apply non-zero equilibrium condition.

\section{Defining the Problem}

The specifications of everted neo-hookean spherical shell are as follows: inner radius A, outer radius B, radial coordinates $\mathrm{R}$ and interval $[\mathrm{A}, \mathrm{B}]$. This spherical shell is very thin with a hole in it and is everted due to the hydrostatic pressure on its outer surface:

$$
r=r(R)
$$

Where $r$ is radial coordinates of a point which is equal to radial coordinates $R$ prior to pressure. After deformation, Inner radius and outer radius are $a_{1}$ and $a_{2}$ respectively and is defined as follows:

$$
a_{1}=\mu_{1} B, a_{2}=\mu_{2} A,
$$

Where $\mu_{1}$ and $\mu_{2}$ are the eigenvalue of the problem. $\mu_{2}$ can be obtained using the following equation:

$$
\mu_{2}=\sqrt[3]{\frac{1+\mu_{1}^{3}}{A_{1}^{3}}}-1
$$

After developing a mathematical model, the problem is changed to a differential equation of order four with the two following boundary conditions:

$$
\begin{gathered}
f^{\prime \prime \prime \prime}+\left(-\frac{8 r^{2}}{c-r^{2}}\right) f^{\prime \prime \prime}+\frac{\left.-c^{2}\left(10+n+n^{2}\right)+2 c\left(2+n+n^{2}\right) r^{3}-2\left(-6+n+n^{2}\right) r^{6}\right)}{\left(r^{2}\left(c-r^{3}\right)^{2}\right)} f^{\prime \prime}+ \\
\frac{2\left(c^{3}\left(10+n+n^{2}\right)-c^{2}\left(14+n+n^{2}\right) r^{3}-3 c\left(-2+n+n^{2}\right) r^{6}+2 n(1+n) r^{9}\right)}{\left(r^{3}\left(c-r^{3}\right)^{3}\right)} f^{\prime}+ \\
\frac{\left(-2+n+n^{2}\right)\left(10 c^{3}-14 c^{2} r^{3}+c n(1+n) r^{6}-n(1+n) r^{9}\right)}{\left(r^{4}\left(c-r^{3}\right)^{3}\right)} f=0, \\
f^{\prime \prime \prime}+\frac{4}{r} f^{\prime \prime}+\frac{\left(-2 c^{2} n(1+n)+4 c n(1+n) r^{3}+(2-3 n(1+n)) r^{6}\right)}{\left(-c r+r^{4}\right)^{2}} f^{\prime} \\
+\left(\frac{2\left(-2+n+n^{2}\right) r^{3}}{\left(c-r^{3}\right)^{2}}\right) f=0, \quad r=a, b, \\
f^{\prime \prime}+\frac{2}{r} f^{\prime}+\left(\frac{\left(-2+n+n^{2}\right)}{r^{2}}\right) f=0, \quad r=a, b,
\end{gathered}
$$

Where $c$ is equals to:

$$
c=a^{3}+B^{3}=b^{3}+A^{3} .
$$

The main stretches of the problem are defined as follows: 


$$
\lambda_{1}=\lambda^{-2}, \lambda_{2}=\lambda, \lambda_{3}=\lambda
$$

$R_{3}$ and $\lambda$ in equation (7) are as follows:

$$
R_{3}=\sqrt[3]{\left(c-r^{3}\right)}, \lambda=\frac{r}{R_{3}}
$$

\section{Applying WKB to Solve the Eigenvalue Problem}

In WKB method the answer is shown as follows:

$$
y(x)=\exp \left[\frac{1}{\delta} \sum_{n=0}^{\infty} \delta^{n} S_{n}(x)\right], \quad \delta>0 .
$$

Where $\delta=\delta(\varepsilon)$ and $\delta \rightarrow 0$. In order to apply WKB method on the everted neo-hookean spherical shell, we define an asymptotic expansion for capital $n$ as follows:

$$
f=\exp \left(n \int S(r) d r\right), S(r)=S_{0}+\frac{1}{n} S_{1}+\frac{1}{n^{2}} S_{2}
$$

Now, by substituting $f$ and its derivatives of equation (8) in equation (3) and considering various coefficients of $n$, we achieve a large class of differential equations in terms of $S(r)$ which could be solved by Mathematica software. Therefore, we can find four independent solutions for $S$ which we show its ith answer as $S^{(i)}$ as follows:

$$
S(r)^{(i)}=S_{0}{ }^{(i)}+\frac{1}{n} S_{1}{ }^{(i)}+\frac{1}{n^{2}} S_{2}{ }^{(i)} .
$$

From which we obtain $\mathrm{S}_{0}{ }^{(\mathrm{i})}, \mathrm{S}_{1}{ }^{(i)}$ and $S_{2}{ }^{(i)}$ as follows:

$$
\begin{aligned}
& \mathrm{S}_{0}{ }^{(1)}=\frac{1}{\mathrm{r}}, \mathrm{S}_{0}{ }^{(2)}=-\frac{1}{\mathrm{r}}, \mathrm{S}_{0}{ }^{(3)}=\frac{\mathrm{r}^{2}}{-\mathrm{c}+\mathrm{r}^{3}}, \mathrm{~S}_{0}{ }^{(4)}=-\frac{\mathrm{r}^{2}}{-\mathrm{c}+\mathrm{r}^{3}}, \\
& \mathrm{~S}_{1}^{(1)}=\frac{c^{2}-c r^{3}+r^{6}}{c^{2} r-3 c r^{4}+2 r^{7}}, \mathrm{~S}_{1}{ }^{(2)}=\frac{2 c^{2}-4 c r^{3}+3 r^{6}}{c^{2} r-3 c r^{4}+2 r^{7}} \\
& S_{1}{ }^{(3)}=\frac{r^{2}\left(c+r^{3}\right)}{c^{2}-3 c r^{3}+2 r^{6}}, S_{1}^{(4)}=\frac{3 r^{5}}{c^{2}-3 c r^{3}+2 r^{6}} \\
& S_{2}{ }^{(1)}=\frac{-6 c^{6}+80 c^{5} r^{3}-183 c^{4} r^{6}}{2 c r\left(c-2 r^{3}\right)^{3}\left(c-r^{3}\right)^{2}}+\frac{-14 c^{3} r^{9}+369 c^{2} r^{12}-262 c r^{15}+20 r^{18}}{2 c r\left(c-2 r^{3}\right)^{3}\left(c-r^{3}\right)^{2}} \text {, } \\
& S_{2}{ }^{(2)}=\frac{6 c^{6}-64 c^{5} r^{3}+263 c^{4} r^{6}-642 c^{3} r^{9}}{2 c r\left(c-2 r^{3}\right)^{3}\left(c-r^{3}\right)^{2}}+\frac{815 c^{2} r^{12}-394 c r^{15}+12 r^{18}}{2 c r\left(c-2 r^{3}\right)^{3}\left(c-r^{3}\right)^{2}} \text {, } \\
& S_{2}{ }^{(3)}=\frac{10 c^{6}-64 c^{5} r^{3}+133 c^{4} r^{6}-162 c^{3} r^{9}}{2 c r^{4}\left(c-2 r^{3}\right)^{3}\left(c-r^{3}\right)}+\frac{379 c^{2} r^{12}-394 c r^{15}-44 r^{18}}{2 c r^{4}\left(c-2 r^{3}\right)^{3}\left(c-r^{3}\right)} \text {, }
\end{aligned}
$$




$$
S_{2}{ }^{(4)}=\frac{-10 c^{6}+64 c^{5} r^{3}-149 c^{4} r^{6}+114 c^{3} r^{9}}{2 c r^{4}\left(c-2 r^{3}\right)^{3}\left(c-r^{3}\right)}+\frac{-11 c^{2} r^{12}+10 c r^{15}-20 r^{18}}{2 c r^{4}\left(c-2 r^{3}\right)^{3}\left(c-r^{3}\right)}
$$

Given that there are four independent solutions for $S(r)$ and considering the definition of $f$ in $(8), f$ has four independent linear solutions and the general solution of $f$ is defined as follows:

$$
f=\sum_{i=1}^{4} k_{i} \exp \left(n \int_{a_{1}}^{r} S^{(i)}(u) d u\right)
$$

Now, we replace equation (12) and its derivatives in (4) and (5) boundary conditions, then write the following matrix:

$$
\sum_{i=1}^{4} \mathrm{c}_{\mathrm{ij}} \mathrm{k}_{\mathrm{j}}=0, \quad(\mathrm{j}=1, \ldots \ldots, 4)
$$

$c_{\mathrm{ij}}$ will be as follows:

$$
c_{\mathrm{ij}}=\left[\begin{array}{rlrl}
\mathrm{F}^{(1)}\left(\mathrm{a}_{1}\right) & \mathrm{F}^{(2)}\left(\mathrm{a}_{1}\right) & \mathrm{F}^{(3)}\left(\mathrm{a}_{1}\right) & \mathrm{F}^{(4)}\left(\mathrm{a}_{1}\right) \\
\mathrm{G}^{(1)}\left(\mathrm{a}_{1}\right) & \mathrm{G}^{(2)}\left(\mathrm{a}_{1}\right) & \mathrm{G}^{(3)}\left(\mathrm{a}_{1}\right) & \mathrm{G}^{(4)}\left(\mathrm{a}_{1}\right) \\
\mathrm{E}^{(1)}\left(\mathrm{a}_{2}\right) \mathrm{F}^{(1)}\left(\mathrm{a}_{2}\right) & \mathrm{E}^{(2)}\left(\mathrm{a}_{2}\right) \mathrm{F}^{(2)}\left(\mathrm{a}_{2}\right) & \mathrm{E}^{(3)}\left(\mathrm{a}_{2}\right) \mathrm{F}^{(3)}\left(\mathrm{a}_{2}\right) & \mathrm{E}^{(4)}\left(\mathrm{a}_{2}\right) \mathrm{F}^{(4)}\left(\mathrm{a}_{2}\right) \\
E^{(1)}\left(\mathrm{a}_{2}\right) \mathrm{G}^{(1)}\left(\mathrm{a}_{2}\right) & \mathrm{E}^{(2)}\left(\mathrm{a}_{2}\right) \mathrm{G}^{(2)}\left(\mathrm{a}_{2}\right) & \mathrm{E}^{(3)}\left(\mathrm{a}_{2}\right) \mathrm{G}^{(3)}\left(\mathrm{a}_{2}\right) & \mathrm{E}^{(4)}\left(\mathrm{a}_{2}\right) \mathrm{G}^{(4)}\left(\mathrm{a}_{2}\right)
\end{array}\right]
$$

Where $\left.E^{(i)}=E^{(i)}(r) g(\mathrm{i}=1, \ldots, 4)\right), E^{(j)} \quad$ constant, $\mathrm{G}^{(\mathrm{j})}(\mathrm{r})$ and $\mathrm{F}^{(j)}(\mathrm{r})$ functions for $j=(1, \ldots, 4)$ are defined as follows:

$$
\begin{gathered}
E^{(j)}(r)=\exp \left(\int_{a_{1}}^{a_{2}} S^{j}(u) d u\right) \\
F^{(j)}(r)=-2+n+n^{2}+n r\left(S^{(j)}(r)\left(2+n r S^{(j)}(r)\right)+r S^{(j)}(r)^{\prime}\right),
\end{gathered}
$$

$$
\frac{\left.-6 c r^{5} S^{(j)}(r) S^{(j)}(r)^{\prime}+3 r^{8} S^{(j)}(r) S^{(j)}(r)^{\prime}\right)}{r^{3}\left(c-r^{3}\right)^{2 / 3}}+\frac{n\left(-2 r^{5}+2 r^{6} S^{(j)}(r)+r\left(c-r^{3}\right)^{2}\left(4 S^{(j)}(r)^{\prime}+r S^{(j)}{ }^{\prime \prime}(r)\right)\right)}{r^{3}\left(c-r^{3}\right)^{2 / 3}}
$$

When $a_{2}-\mathrm{a}_{1}=\mathrm{O}(1)$ and $A_{1}-1=\mathrm{O}(1)$, for capital value $n E^{(1)}$ and $E^{(3)}$ with capital exponent and small $E^{(2)}$ and $E^{(4)}$. To obtain a non-trivial solution of (13) the following condition must be satisfied: 


$$
\operatorname{det}\left(c_{i j}\right)=0
$$

Then,

$$
\left|\begin{array}{ll}
F^{(1)}\left(a_{2}\right) & F^{(3)}\left(a_{2}\right) \\
G^{(1)}\left(a_{2}\right) & G^{(3)}\left(a_{2}\right)
\end{array}\right|=0,
$$

Now we expand $\mu_{1}$ in terms of $n$ and thus we consider:

$$
\mu_{1}=c_{0}+\frac{1}{n} c_{1}+\frac{1}{n^{2}} c_{2}
$$

Now, using Mathematica software and considering various coefficients of $n, \mu_{1}$ can be obtained as follows:

$$
\mu_{1}=0.669096+\frac{1}{n} 0.020921-\frac{1}{n^{2}} 7.711126
$$

Table 1 shows the values obtained by applying WKB method using expansion of $S(r)=\sum_{i=0}^{2} \frac{1}{n^{2}} S_{i}$ for various $n \mathrm{~s}$.

Table 1. Values obtained by applying WKB method using expansion of $S(r)=\sum_{i=0}^{2} \frac{1}{n^{2}} S_{i}$ for various $n s$

\begin{tabular}{|l|l|l|l|}
\hline mode & \multicolumn{1}{|c|}{$\mathrm{A}_{1}(\mathrm{wkb})$} & \multicolumn{1}{|c|}{$\mu_{1}(\mathrm{wkb})$} & \multicolumn{1}{|c|}{$\mu_{2}(\mathrm{wkb})$} \\
\hline 25 & 0.199658 & 0.657595 & 5.43305 \\
\hline 50 & 0.253889 & 0.66643 & 4.27612 \\
\hline 100 & 0.266193 & 0.66877 & 4.07879 \\
\hline 150 & 0.268268 & 0.668893 & 4.04112 \\
\hline 200 & 0.268933 & 0.669056 & 4.0373 \\
\hline 300 & 0.269349 & 0.66908 & 4.03106 \\
\hline 400 & 0.269465 & 0.6691 & 4.02933 \\
\hline
\end{tabular}

Solving everted neo-hookean spherical shell problem using WKB method would be possible through defining the asymptotic expansion below:

$$
f(r)=R(r) \exp \left(n \int S(r) d r\right), R(r)=R_{0}+\frac{1}{n} R_{1}+\frac{1}{n^{2}} R_{2} .
$$

By substituting (22) and its relevant derivatives in problem with eigenvalue (3), we apply WKB method and obtain $S, R_{0}, R_{1}$ and $R_{2}$ as follows:

$$
S(r)^{(1)}=\frac{1}{r}, \mathrm{~S}(\mathrm{r})^{(2)}=-\frac{1}{r}, \mathrm{~S}(\mathrm{r})^{(3)}=\frac{r^{2}}{-c+r^{3}}, \mathrm{~S}(\mathrm{r})^{(4)}=-\frac{r^{2}}{-c+r^{3}}
$$

Using Mathematica software and substituting $n^{3}$ in equation (3) and substituting relation (23), four distinct $R_{0}$ are obtained as follows: 


$$
R_{0}^{(1)}=\frac{r^{2}\left(c-r^{3}\right)^{1 / 3}}{\sqrt{c-2 r^{3}}}, R_{0}^{(2)}=-\frac{r\left(c-r^{3}\right)^{1 / 3}}{\sqrt{c-2 r^{3}}}, R_{0}^{(3)}=\frac{\left(c-r^{3}\right)^{2 / 3}}{\sqrt{c-2 r^{3}}}, R_{0}{ }^{(4)}=\frac{\left(c-r^{3}\right)}{\sqrt{c-2 r^{3}}}
$$

At this stage, by substituting $\mathrm{n}^{2}$ in $(3), R_{1}{ }^{(\mathrm{i})}$ for $(i=1, \ldots, 4)$ and using Mathematica software we will have:

$$
\begin{aligned}
& \mathrm{R}_{1}{ }^{(1)}=\frac{\mathrm{r}^{2}\left(\mathrm{c}-\mathrm{r}^{3}\right)^{1 / 3}\left(\frac{5 \mathrm{r}^{3}}{6 \mathrm{c}}-\frac{9 \mathrm{c}^{2}}{8\left(\mathrm{c}-2 \mathrm{r}^{3}\right)^{2}}-\frac{c\left(c+7 r^{3}\right)}{6\left(c^{2}-3 \mathrm{c} \mathrm{r}^{3}+2 \mathrm{r}^{6}\right)}\right.}{2 \sqrt{\mathrm{c}-2 \mathrm{r}^{3}}} \\
& +\frac{\frac{2}{3} \log \left(-c+r^{3}\right)+\log \left(\left(-c+2 r^{3}\right)\right)}{2 \sqrt{c-2 r^{3}}} \\
& \mathrm{R}_{1}{ }^{(2)}=\frac{\mathrm{r}\left(31 \mathrm{c}^{4}-27 \mathrm{c}^{3} \mathrm{r}^{3}+44 \mathrm{c}^{2} \mathrm{r}^{6}-160 \mathrm{c} \mathrm{r}^{9}+80 \mathrm{r}^{12}\right.}{48 \mathrm{c}\left(\mathrm{c}-2 \mathrm{r}^{3}\right)^{5 / 2}\left(\mathrm{c}-\mathrm{r}^{3}\right)^{3}} \\
& -\frac{\left.8 c\left(c-2 r^{3}\right)^{2}\left(c-r^{3}\right)\left(2 \log \left(-c+r^{3}\right)+3 \log \left(-c+2 r^{3}\right)\right)\right)}{48 c\left(c-2 r^{3}\right)^{5 / 2}\left(c-r^{3}\right)^{2 / 3}}, \\
& \mathrm{R}_{1}{ }^{(3)}=\frac{1}{48 c \mathrm{r}^{3}\left(\mathrm{c}-2 \mathrm{r}^{3}\right)^{5 / 2}}\left(\mathrm{c}-\mathrm{r}^{3}\right)^{2 / 3}\left(-80 \mathrm{c}^{4}+311 \mathrm{c}^{3} \mathrm{r}^{3}-228 \mathrm{c}^{2} \mathrm{r}^{6}-80 \mathrm{cr} \mathrm{r}^{9}+\right. \\
& \left.80 r^{12}+8 c r^{3}\left(c-2 r^{3}\right)^{2}\left(18 \log (r)+2 \log \left(-c+r^{3}\right)-3 \log \left(-c+2 r^{3}\right)\right)\right), \\
& R_{1}{ }^{(4)}=\frac{1}{48 c r^{3}\left(c-2 r^{3}\right)^{5 / 2}}\left(c-r^{3}\right)\left(80 c^{4}-311 c^{3} r^{3}+228 c^{2} r^{6}+80 c r^{9}-80 r^{12}\right. \\
& +8 \mathrm{cr}^{3}\left(\mathrm{c}-2 \mathrm{r}^{3}\right)^{2}\left(-2\left(9 \log (\mathrm{r})+\log \left(-\mathrm{c}+\mathrm{r}^{3}\right)\right)+3 \log \left(-\mathrm{c}+2 \mathrm{r}^{3}\right)\right) .
\end{aligned}
$$

Now we write the obtained results of $R_{0}{ }^{(\mathrm{i})}$ and $R_{1}{ }^{(\mathrm{i})}$ as (22) expansion, so that in this expansion we suppose $R_{2}{ }^{(\mathrm{i})}(\mathrm{r})$ as x for $(i=1, \ldots, 4)$ and in next stages try to eliminate it to find integer of $R^{(i)}(r)$ :

$$
R^{(i)}(r)=R_{0}{ }^{(i)}+\frac{1}{n} R_{1}{ }^{(i)}+R_{2}{ }^{(i)}(r)
$$

Since there are four distinct $R(r)$ and considering the definition of $f$ in (22), there are four independent solutions for $f$ which its general solutions will be as follows:

$$
f=\sum_{i=1}^{4} k_{i} R(r) \exp \left(n \int_{a_{1}}^{r} S^{(j)}(u) d u\right), \quad(j=1, \ldots, 4),
$$

We substitute equation (26) and its derivatives in (4) and (5) boundary condition and write it as the matrix below:

$$
\sum_{i=1}^{4} \mathrm{c}_{\mathrm{ij}} \mathrm{k}_{\mathrm{j}}=0, \quad(\mathrm{j}=1, \ldots \ldots, 4) .
$$

$c_{\mathrm{ij}}$ is defined as follows: 


$$
c_{\mathrm{ij}}=\left[\begin{array}{rlrl}
\alpha^{(1)}\left(\mathrm{a}_{1}\right) & \alpha^{(2)}\left(\mathrm{a}_{1}\right) & \alpha^{(3)}\left(\mathrm{a}_{1}\right) & \alpha^{(4)}\left(\mathrm{a}_{1}\right) \\
\gamma^{(1)}\left(\mathrm{a}_{1}\right) & \gamma^{(2)}\left(\mathrm{a}_{1}\right) & \gamma^{(3)}\left(\mathrm{a}_{1}\right) & \gamma^{(4)}\left(\mathrm{a}_{1}\right) \\
\mathrm{E}^{(1)}\left(\mathrm{a}_{2}\right) \alpha^{(1)}\left(\mathrm{a}_{2}\right) & \mathrm{E}^{(2)}\left(\mathrm{a}_{2}\right) \alpha^{(2)}\left(\mathrm{a}_{2}\right) & \mathrm{E}^{(3)}\left(\mathrm{a}_{2}\right) \alpha^{(3)}\left(\mathrm{a}_{2}\right) & \mathrm{E}^{(4)}\left(\mathrm{a}_{2}\right) \alpha^{(4)}\left(\mathrm{a}_{2}\right) \\
\mathrm{E}^{(1)}\left(\mathrm{a}_{2}\right) \gamma^{(1)}\left(\mathrm{a}_{2}\right) & \mathrm{E}^{(2)}\left(\mathrm{a}_{2}\right) \gamma^{(2)}\left(\mathrm{a}_{2}\right) & \mathrm{E}^{(3)}\left(\mathrm{a}_{2}\right) \gamma^{(3)}\left(\mathrm{a}_{2}\right) & \mathrm{E}^{(4)}\left(\mathrm{a}_{2}\right) \gamma^{(4)}\left(\mathrm{a}_{2}\right)
\end{array}\right]
$$

Where $E^{(i)}=E^{(i)}(r)(\mathrm{i}=1, \ldots, 4)$, constant $E^{(j)}$ and $\gamma^{(\mathrm{j})}(\mathrm{r})$ and $\alpha^{(j)}(\mathrm{r})$ functions for $j=(1, \ldots, 4)$ is defined as follows:

$$
\begin{gathered}
E^{(j)}=\exp \left(n \int_{a_{1}}^{a_{2}} S^{j}(u) d u\right), \\
\alpha^{(j)}(\mathrm{r})=\alpha_{1}^{(j)}+\frac{1}{n} \alpha_{2}^{(j)}+\frac{1}{n^{2}} \alpha_{3}^{(j)}, \\
\gamma^{(j)}(\mathrm{r})=\gamma_{1}^{(j)}+\frac{1}{n} \gamma_{2}{ }^{(j)}+\frac{1}{n^{2}} \gamma_{3}^{(j)},
\end{gathered}
$$

Now at this stage, by substituting $R^{(\mathrm{i})}(r)$ for $(i=1, \ldots, 4)$ in (4) and (5) boundary conditions, we obtain $\gamma^{(j)}(\mathrm{r})$ and $\gamma^{(j)}(\mathrm{r})$ respectively. We know for capital value $E^{(1)}$ and $E^{(3)}$ with capital exponent and small $E^{(2)}$ and $E^{(4)}$. In order to obtain nontrivial solution for equation (27) the following condition must be satisfied:

$$
\operatorname{det}\left(c_{i j}\right)=0
$$

Therefore, we have:

$$
\left|\begin{array}{ll}
\alpha^{(1)}\left(\mathrm{a}_{2}\right) & \alpha^{(3)}\left(\mathrm{a}_{2}\right) \\
\gamma^{(1)}\left(\mathrm{a}_{2}\right) & \gamma^{(3)}\left(\mathrm{a}_{2}\right)
\end{array}\right|=0
$$

The matrix equation below can be substituted by matrix equation (27):

$$
\left[\begin{array}{ll}
\alpha^{(1)}\left(\mathrm{a}_{2}\right) & \alpha^{(3)}\left(\mathrm{a}_{2}\right) \\
\gamma^{(1)}\left(\mathrm{a}_{2}\right) & \gamma^{(3)}\left(\mathrm{a}_{2}\right)
\end{array}\right]\left[\begin{array}{l}
k_{1} \\
\mathrm{k}_{3}
\end{array}\right]=\left[\begin{array}{l}
0 \\
0
\end{array}\right]
$$

In order to eliminate $R_{2}{ }^{(\mathrm{i})}(\mathrm{r})$ for $(i=1, \ldots, 4)$ of matrix (34) elements, we write this equation as follows:

$$
\left[\begin{array}{ll}
\alpha^{\left(\widehat{1)}\left(\mathrm{a}_{2}\right)\right.} & \alpha^{\left(\widehat{3)}\left(\mathrm{a}_{2}\right)\right.} \\
\gamma^{\left(\widehat{1)}\left(\mathrm{a}_{2}\right)\right.} & \gamma^{\left(\widehat{3)}\left(\mathrm{a}_{2}\right)\right.}
\end{array}\right]\left[\begin{array}{l}
k_{1} \mathrm{R}^{(1)}\left(\mathrm{a}_{2}\right) \\
\mathrm{k}_{3} \mathrm{R}^{(3)}\left(\mathrm{a}_{2}\right)
\end{array}\right]=\left[\begin{array}{l}
0 \\
0
\end{array}\right]
$$

Where

$$
\begin{aligned}
& \left\{\alpha^{\left(\widehat{3)}\left(\mathrm{a}_{2}\right)\right.}, \gamma^{\left(\widehat{3)}\left(\mathrm{a}_{2}\right)\right.}\right\}=\left\{\alpha^{(3)}\left(\mathrm{a}_{2}\right), \gamma^{(3)}\left(\mathrm{a}_{2}\right)\right\} / R^{(3)}(r), \\
& \left\{\alpha^{\left(\widehat{1)}\left(\mathrm{a}_{2}\right)\right.}, \gamma^{\left(\widehat{1)}\left(\mathrm{a}_{2}\right)\right.}\right\}=\left\{\alpha^{(1)}\left(\mathrm{a}_{2}\right), \gamma^{(1)}\left(\mathrm{a}_{2}\right)\right\} / R^{(1)}(r),
\end{aligned}
$$


Matrix (35) will have a solution if determinant of the coefficient matrix is zero:

$$
\left|\begin{array}{ll}
\alpha^{\left(\widehat{1)}\left(\mathrm{a}_{2}\right)\right.} & \alpha^{\left(\widehat{3)}\left(\mathrm{a}_{2}\right)\right.} \\
\gamma^{\left(\widehat{1)}\left(\mathrm{a}_{2}\right)\right.} & \gamma^{\left(\widehat{3)}\left(\mathrm{a}_{2}\right)\right.}
\end{array}\right|=0,
$$

Now given equation (20), $C_{0}, C_{1}$ and $C_{2}$ will be as follows:

$$
\mathrm{C}_{0}=0.66614233, \quad \mathrm{C}_{1}=1.37704, \quad \mathrm{C}_{2}=-23.2898 .
$$

By substituting (38) in (20) we have the equation bellow:

$$
\mu_{1}=0.66614233+\frac{1}{n} 1.37704-\frac{1}{n^{2}}-23.2898
$$

\subsection{The Equilibrium Condition}

The shell is composed of a homogeneous, isotopic and hyper elastic material, and the strain-energy function is in relation with deformation gradient due to principle stretches and is written:

$$
w=w\left(\lambda_{1}, \lambda_{2}, \lambda_{3}\right)
$$

Now stretch tensors may be written:

$$
\sigma_{i i}=\sigma_{\mathrm{i}}-\mathrm{p}, \quad \mathrm{i}=1,2,3
$$

Where $p$ is pressure and $w_{\mathrm{i}}=\frac{\delta \mathrm{w}}{\delta \lambda_{i}}$. The strain-energy function of the everted neo-hookean spherical shell is:

$$
w=\mu\left(\lambda_{1}^{2}+\lambda_{2}^{2}+\lambda_{3}^{2}-3\right)
$$

Now we check the only equilibrium condition:

$$
\frac{d}{\mathrm{dr}} \sigma_{11}+\frac{2}{\mathrm{r}}\left(\sigma_{11}-\sigma_{22}\right)=0
$$

We assume that the spherical shell remains empty after deformation, then $a>0$. Also, if the stretch on inner and outer surface is zero, it can be written:

Now the equilibrium mentioned in equation (41) may be written:

$$
\sigma_{11}(r)=2 \int_{b}^{\mathrm{r}} \frac{\sigma_{22}-\sigma_{11}}{\mathrm{r}} \mathrm{dr}
$$

Where $b=r(A)=a^{3}+B^{3}-A^{3}$. The principle stretches are defined as follows:

$$
\lambda_{1}=-r^{\prime}(\mathrm{R})=\frac{\delta \mathrm{r}}{\delta \mathrm{R}}, \quad \lambda_{2}=\lambda_{3}=\frac{\mathrm{r}}{\mathrm{R}}
$$

Based on the incompressibility condition we have: 


$$
\lambda_{1} \lambda_{2} \lambda_{3}=1
$$

By assuming $\lambda_{2}=\lambda_{3}=\lambda$ we can obtain the following result for $\lambda_{1}$ :

$$
\lambda_{1}=\lambda^{-2}
$$

Therefore based on (40) and (44) the energy function of the problem may be written:

By assuming $\mu=1$ we can obtain the equation bellow:

$$
\lambda \widehat{W_{\lambda}}=\lambda \frac{\mathrm{d} \widehat{W}}{\mathrm{~d} \lambda}=2\left(\sigma_{22}-\sigma_{11}\right)
$$

At this stage, using Mathematica software, equation (42) may be written:

$$
\begin{gathered}
\sigma_{11}(r)=-\int_{\lambda_{\mathrm{r}}}^{\lambda_{\mathrm{b}}} \frac{\widehat{W}_{\lambda}(\lambda)}{\left(1+\lambda^{3}\right)} \mathrm{d} \lambda=-2 \int_{\lambda_{\mathrm{r}}}^{\lambda_{\mathrm{b}}} \frac{\left(\lambda^{2}-\lambda^{-4}\right)}{\lambda\left(1+\lambda^{3}\right)} \mathrm{d} \lambda \\
=-\frac{2 \mathrm{~B}}{\mathrm{a}}-\frac{\mathrm{B}^{4}}{2 \mathrm{a}^{4}}-\frac{\mathrm{A}^{4}}{2\left(\mathrm{a}^{3}-\mathrm{A}^{3}+\mathrm{B}^{3}\right)^{4 / 3}}+\frac{2 \mathrm{~A}}{\left(\mathrm{a}^{3}-\mathrm{A}^{3}+\mathrm{B}^{3}\right)^{1 / 3}}, \\
\lambda_{\mathrm{b}}=\frac{\mathrm{r}}{\left(\mathrm{a}^{3}+\mathrm{B}^{3}-\mathrm{r}^{3}\right)^{1 / 3}}, \lambda_{\mathrm{r}}=\frac{\left(\mathrm{a}^{3}-\mathrm{A}^{3}+\mathrm{B}^{3}\right)^{1 / 3}}{\mathrm{~A}} .
\end{gathered}
$$

By drawing the graph of equilibrium condition and its collision with the graph resulted from applying WKB method, the desired solutions for 2 arbitrary $n$ are shown in figure (1):

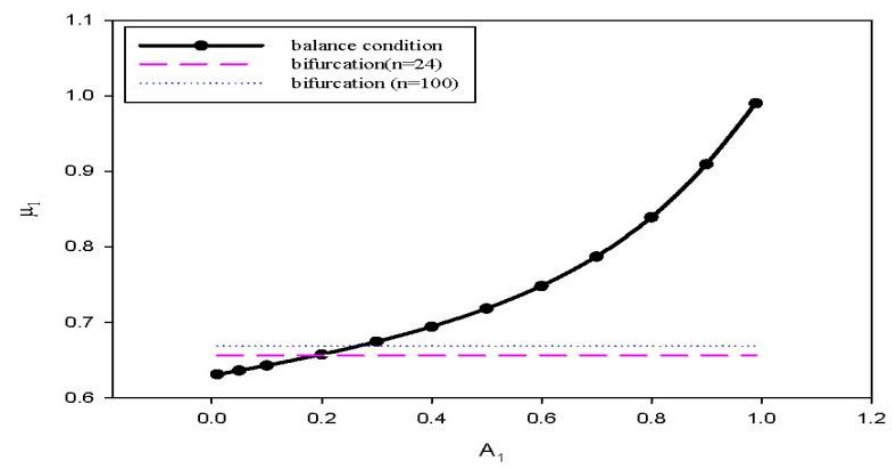

Figure 1. collision of bifurcation graph and the equilibrium condition graph of everted spherical shell for two arbitrary $n, n=24$ and $n=100$.

In figure 1, we find the solution for $n=24$ and $n=100$ by drawing the graph of equation (39) and (46) in the neighborhood of point $A_{1}=0.2$. Now in table 2, using the equilibrium condition on (46) we explain the eigenvalues obtained from WKB method via $R(r)$ expansion. It should be noted that all the calculations in this method have been carried out by means of Mathematica software. 
Table 2- numbers obtained by means of WKB and using $R(r)=\sum_{i=0}^{2} \frac{1}{n^{i}} R_{i}$ expansion for equation (2) of order four

\begin{tabular}{|c|c|c|c|}
\hline mode & $A_{1}(w k b)$ & $\mu_{1}(w k b)$ & $\mu_{2}(w k b)$ \\
\hline 25 & 0.164156 & 0.68396 & 7.98636 \\
\hline 50 & 0.21247 & 0.684367 & 5.15105 \\
\hline 100 & 0.245256 & 0.677584 & 4.44583 \\
\hline 150 & 0.255752 & 0.674288 & 4.25625 \\
\hline 200 & 0.260942 & 0.672445 & 4.16776 \\
\hline 300 & 0.266103 & 0.670474 & 4.08297 \\
\hline 400 & 0.268674 & 0.669439 & 4.041186 \\
\hline
\end{tabular}

\subsection{The Turning Point}

According to $\mathrm{Fu}$ and $\mathrm{Lin}$ (2000) [10], turning point is the root of $R_{0}$ denominator in WKB method by means of $R(r)=\sum_{i=0}^{2} \frac{1}{n^{i}} R_{i}$ expansion. They believe that turning point could also be achieved by $S_{0}{ }^{(2)}=S_{0}{ }^{(4)}$. In this paper, after finding the turning point, the solution is obtained within a certain interval.

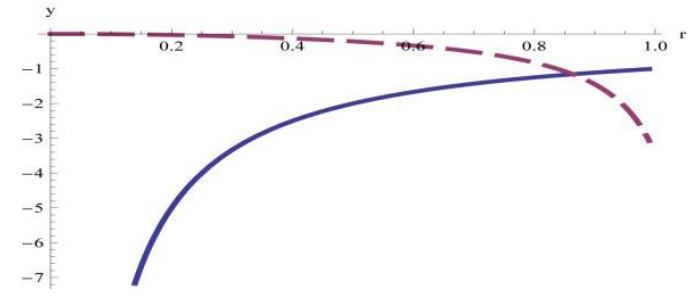

Figure 2. turning point of $S_{0}^{(4)}$ and $S_{0}{ }^{(2)}$

Now by studying the root of denominator $R_{0}$, the following statement is obtained:

By studying the obtained $S_{0}$ and $S_{0}{ }^{(2)}=S_{0}{ }^{(4)}$, we reach the same conclusion.

$$
\frac{-1}{r}=\frac{\mathrm{r}^{2}}{-\mathrm{c}+\mathrm{r}^{3}} \rightarrow \mathrm{r}=\sqrt[3]{\frac{\mathrm{c}}{2}}
$$

Now for a constant $n$, we calculate $\mu_{1}$ and by substituting it in the constant $C$ defined in equation (6), we have:

$$
C=a^{3}+B^{3}=b^{3}+A^{3}=\mu_{1}^{3}+B^{3}=\mu_{1}{ }^{3}+1
$$


At this stage, by substituting (48) in (47) we may obtain the turning point. Therefore, using expansions $S(r)$ and $R(r)$ of WKB method, we calculate the turning point for $n=30$ and name them $r_{\mathrm{R}}$ and $r_{\mathrm{S}}$ respectively.

$$
r_{\mathrm{s}}=0.863811, \quad r_{\mathrm{R}}=0.871332 .
$$

\section{Applying Numerical Compound Matrix in Solving the Eigenvalue Problem}

In numerical compound method, we first define a matrix solution with certain initial conditions and then obtain the eignevallues as the root of matrix cofactors. Compound matrix variables are the matrix solution cofactors and in this method, cofactors are calculated directly.

We suppose the eigenvalue problem below:

$$
L(\varphi)=\varphi^{(4)}-a_{1} \varphi^{(3)}-a_{2} \varphi^{(2)}-a_{3} \varphi^{\prime}-a_{4} \varphi,
$$

Which in boundary condition becomes:

$$
\left\{\begin{array}{cc}
\varphi^{(3)}-\alpha_{1} \varphi^{(2)}-\alpha_{2} \varphi^{\prime}-\alpha_{3} \varphi=0, & r=a, b \\
\varphi^{(2)}-\beta_{1} \varphi^{\prime}-\beta_{2} \varphi=0, & \quad r=a, b
\end{array}\right.
$$

We rewrite equation (49) of order four and its boundary condition as follows:

$$
\begin{gathered}
\mathrm{A}(\mathrm{x})=\left(\begin{array}{cccc}
0 & 1 & 0 & 0 \\
0 & 0 & 1 & 0 \\
0 & 0 & 0 & 1 \\
\mathrm{a}_{4} & \mathrm{a}_{3} & \mathrm{a}_{2} & \mathrm{a}_{1}
\end{array}\right), \\
\varphi^{\prime}=A(x) \varphi, \varphi=\left[\varphi, \varphi^{\prime}, \varphi^{\prime \prime}, \varphi^{\prime \prime \prime}\right], \\
C(x) V=0, \quad C(x)=\left(\begin{array}{cccc}
\alpha_{3} & \alpha_{2} & \alpha_{1} & 1 \\
\beta_{2} & \beta_{1} & 1 & 0
\end{array}\right),
\end{gathered}
$$

The general solution of equation (51) under initial condition may be written

$$
\varphi=\alpha \varphi_{1}+\beta \varphi_{2}
$$

Where $\varphi_{1}$ and $\varphi_{2}$ are two independent linear solutions, obtained from the homogenous system which satisfies the initial condition below:

$$
\varphi_{1}=\left[\begin{array}{llll}
0 & 0 & 1 & 0
\end{array}\right]^{T}, \varphi_{2}=\left[\begin{array}{llll}
0 & 0 & 0 & 1
\end{array}\right]^{T},
$$

The first step of the numerical compound matrix is based on the matrix solution cofactors which may be written:

$$
\phi=\left[\begin{array}{ll}
\varphi_{1} & \varphi_{2} \\
\varphi_{1}{ }^{\prime} & \varphi_{2}{ }^{\prime \prime} \\
\varphi_{1}^{\prime \prime} & \varphi_{2}{ }^{\prime \prime \prime} \\
\varphi_{1}{ }^{\prime \prime} & \varphi_{2}{ }^{\prime \prime}
\end{array}\right]
$$


The cofactors of matrix solution may be as shown as follows:

$$
\begin{gathered}
y_{1}=\left|\begin{array}{ll}
\varphi_{1} & \varphi_{2} \\
\varphi_{1}^{\prime} & \varphi_{2}
\end{array}\right|, y_{2}=\left|\begin{array}{cc}
\varphi_{1} & \varphi_{2} \\
\varphi_{1}^{\prime \prime} & \varphi_{2}^{\prime \prime}
\end{array}\right|, y_{3}=\left|\begin{array}{cc}
\varphi_{1} & \varphi_{2} \\
\varphi_{1}{ }^{\prime \prime \prime} & \varphi_{2}{ }^{\prime \prime \prime}
\end{array}\right|, \\
y_{4}=\left|\begin{array}{ll}
\varphi_{1}^{\prime} & \varphi_{2}^{\prime} \\
\varphi_{1}^{\prime \prime} & \varphi_{2}^{\prime \prime}
\end{array}\right|, \quad y_{5}=\left|\begin{array}{cc}
\varphi_{1}^{\prime} & \varphi_{2}^{\prime} \\
\varphi_{1}^{\prime \prime \prime} & \varphi_{2}^{\prime \prime \prime}
\end{array}\right|, \quad y_{6}=\left|\begin{array}{ll}
\varphi_{1}^{\prime \prime} & \varphi_{2}^{\prime \prime} \\
\varphi_{1}^{\prime \prime \prime} & \varphi_{2}^{\prime \prime \prime}
\end{array}\right| .
\end{gathered}
$$

We know that $y=\left[y_{1}, \ldots, y_{6}\right]^{T}$ is $\varphi$ compound of order two. Now by differentiating the cofactors of matrix solution, we may write problem (49) using the numerical compound matrix method [12] as follows:

$$
y^{\prime}=f(r, y)=\left[\begin{array}{c}
y_{2} \\
y_{3}+y_{4} \\
a_{3} y_{1}+a_{2} y_{2}+a_{1} y_{3}+y_{5} \\
y_{5} \\
-a_{4} y_{1}+a_{2} y_{4}+a_{1} y_{5} y_{6} \\
-a_{4} y_{2}-a_{3} y_{4}+a_{1} y_{6}
\end{array}\right], \quad a \leq r \leq b
$$

Now to obtain the initial condition of (53) compound problem, we substitute $\varphi_{1}$ and $\varphi_{2}$ in (50) boundary condition and considering the compound variables obtained in matrix (53), the initial condition of compound problem may be written $6 \times 1$ :

$$
y(a)=\left[\begin{array}{c}
\mathrm{y}_{1}(\mathrm{a}) \\
-\beta_{1} \mathrm{y}_{1}(\mathrm{a}) \\
\left(\alpha_{1} \beta_{1}-\alpha_{2}\right) \mathrm{y}_{1}(\mathrm{a}) \\
\beta_{2} \mathrm{y}_{1}(\mathrm{a}) \\
\left(\alpha_{3}-\alpha_{1} \beta_{2}\right) \mathrm{y}_{1}(\mathrm{a}) \\
\left.\alpha_{2} \beta_{2}-\alpha_{3} \beta_{1}\right) \mathrm{y}_{1}(\mathrm{a})
\end{array}\right]
$$

We know $\varphi=\alpha \varphi_{1}+\beta \varphi_{2}$. By substituting $\varphi$ in (50) we have:

$$
\left[\begin{array}{cc}
\varphi_{1}{ }^{(3)}-\alpha_{1} \varphi_{1}{ }^{(2)}-\alpha_{2} \varphi_{1}{ }^{\prime}-\alpha_{3} \varphi_{1} & \varphi_{2}{ }^{(3)}-\alpha_{1} \varphi_{2}{ }^{(2)}-\alpha_{2} \varphi_{2}{ }^{\prime}-\alpha_{3} \varphi_{2} \\
\varphi_{1}{ }^{(2)}-\beta_{1} \varphi_{1}{ }^{\prime}-\beta_{2} \varphi_{1} & \varphi_{2}{ }^{(2)}-\beta_{1} \varphi_{2}{ }^{\prime}-\beta_{2} \varphi_{2}
\end{array}\right]\left[\begin{array}{l}
c_{1} \\
c_{2}
\end{array}\right]=0
$$

Equation (55) will have non-trivial solution, if the determinant of the coefficient matrix is zero:

$$
\left|\begin{array}{cc}
\varphi_{1}{ }^{(3)}-\alpha_{1} \varphi_{1}{ }^{(2)}-\alpha_{2} \varphi_{1}{ }^{\prime}-\alpha_{3} \varphi_{1} & \varphi_{2}{ }^{(3)}-\alpha_{1} \varphi_{2}{ }^{(2)}-\alpha_{2} \varphi_{2}{ }^{\prime}-\alpha_{3} \varphi_{2} \\
\varphi_{1}{ }^{(2)}-\beta_{1} \varphi_{1}{ }^{\prime}-\beta_{2} \varphi_{1} & \varphi_{2}{ }^{(2)}-\beta_{1} \varphi_{2}{ }^{\prime}-\beta_{2} \varphi_{2}
\end{array}\right|=0 .
$$

Therefore, the target condition is realized as follows:

$$
\begin{gathered}
\left(\alpha_{2} \beta_{2}-\alpha_{3} \beta_{1}\right) \mathrm{y}_{1}(\mathrm{~b})+\left(\alpha_{1} \beta_{2}-\alpha_{3}\right) \mathrm{y}_{2}(\mathrm{~b})+\beta_{2} \mathrm{y}_{3}(\mathrm{~b})+\left(\alpha_{1} \beta_{1}-\alpha_{2}\right) \mathrm{y}_{4}(\mathrm{~b}) \\
+\beta_{1} \mathrm{y}_{5}(\mathrm{~b})+\mathrm{y}_{6}(\mathrm{~b})=0 .
\end{gathered}
$$

At this stage, we solve the initial value (57) using Fortran programming language. Here we apply RungeKutta-Fehlberg numerical method [12] and define $k_{\mathrm{i}}(i=1,2, \ldots, 6)$ as six-elements vectors and finally 
estimate $y_{\mathrm{i}}$ i.e. compound variables in point $r=b$. After that by substituting the obtained $y_{\mathrm{i}}$ in target condition, the eigenvalue $\mu$ is achieved. Therefore, using Newton or bisection method [12] we obtain $\mu$.

Table 3. numbers obtained using numerical compound method for various $\boldsymbol{n}$ for equation of order four

\begin{tabular}{|c|c|c|c|}
\hline mode & $A_{1}$ (compound) & $\mu_{1}$ (compound) & $\mu_{2}$ (compound) \\
\hline 25 & 0.4451957 & 0.7034549 & 2.425999 \\
\hline 50 & 3.544032 & 6.848799 & 3.061043 \\
\hline 100 & 0.3021996 & 0.6749331 & 3.592747 \\
\hline 150 & 0.2856226 & 0.6719459 & 3.801494 \\
\hline 200 & 0.2761169 & 0.6702654 & 3.932344 \\
\hline 300 & 0.2660719 & 0.6685150 & 4.080656 \\
\hline 400 & 0.2615474 & 0.6677353 & 4.151138 \\
\hline
\end{tabular}

\section{Results}

In WKB method, the solution is described as a linear combination of exponential power series of small parameter $\delta$ :

$$
y(x)=\exp \left[\frac{1}{\delta} \sum_{n=0}^{\infty} \delta^{n} S_{n}(x)\right], \quad \delta>0 .
$$

Where $\delta=\delta(\varepsilon)$ and $\delta \rightarrow 0$. In the present paper, we applied WKB method on the problem of everted neohookean spherical shell using expansions $\mathrm{S}(\mathrm{r})=\sum_{\mathrm{i}=0}^{2} \frac{1}{\mathrm{n}^{\mathrm{i}}} \mathrm{S}_{\mathrm{i}}$ and $R(r)=\sum_{i=0}^{2} \frac{1}{n^{2}} R_{i}$ and by comparing the data obtained by applying these two expansions and WKB method, particularly for small $n$, concluded that they didn't match due to a turning point in interval $[1,0]$. By applying the numerical compound matrix method on the above mentioned problem and comparing the data obtained from this method and WKB method, we conclude that the turning point causes data mismatch.

\section{Reference}

[1] B.S. Ng, W.H. Reid, A Numerical Method for Linear Two-point Boundary-value Problems Using Compound Matrices, Journal of Computational Physics, vol.30, pp.70-85,1979.

[2] B.S. Ng, W.H. Reid, An Initial Value Matrix Method for Eigen-value Problems Using Compound Matrices, Journal of Computational Physics, vol.30, pp. 125-136,1979.

[3] B.S. Ng, W.H. Reid, The Compound Matrix Method for Ordinary Differential Systems, Journal of Computational Physics, vol. 58, pp. 209-228, 2985.

[4] Chen Yi-Chao and Haughton D.M., On the Existence of Elastic Cylinders, .Elasticity 49, 79-88, 1997.

[5] Fu. Y.B and Sanjaranipour M., WKB Method with Repeated Roots and its Application to the Buckling Analysis of an Everted Cylindrical Tube, SIAM Journal of Applied Mathematics 62 1876, 2002.

[6] Fu Y.B, Some Asymptotic Results Concern the Buckling of Arbitrary Thickness, int. J. Non -linear Mech. 33,11111112,1978 .

[7] Houghton D.M and Chen Yi-Chao, On the Eversion of Incompressible Elastic Spherical Shells, ZAMP 50, 312-326, 1999.

[8] Haughton D.M, and Chen Yi-Chao, Asymptotic Bifurcation for the Eversion of Elastic Shells, ZAMP 54, 191-211, 2003.

[9] Sanjaranipour M., WKB Analysis of the Buckling of a Neo-hookean Cylindrical Shell of Arbitrary Thickness Subjected to an External Pressure, International Journal of Applied Mathematics, Vol, no. 4(2010)857-870.

[10] Sanjaranipour M., Application of WKB Method to Some of the Buckling Problems in Finite Elasticity, Ph. D. thesis Keele.2001. 
[11] Fu Y.B, Lin Y. P, A WKB Analysis of the Buckling of an Everted Neo-Hookean Cylindrical Tube, Department of Mathematics, Kunming Institute of Science and Technology, Kunming, China.

[12] Richard L. Borden, J. Douglas Faires, Albert C. Reynolds, Numerical Analysis, Ali Akbar Alamzadeh, Esmaeil Babelian, Mohammad Reza Omidvar, Qoqnoos Publication, Tehran, 2000.

[13] Bush A.W., Protection for Engineers and Scientists, School of Computing and Mathematics Teesside, Polytechnic Middleborough, U. k, 1992.

[14] Nyfeh, Alihassan, Introduction to Perturbation Technique, University Distinguished Professor Virginia Polytechnic and University of Blackbury Virginia and Yamourk University Irbid Jordan, April 1980.

[15]Carl M. Bender, Advanced Mathematical Methods for Scientists and Engineers, copy- right 1978 by McGraw-Hill Inc.

[16] Chadwick P., Continuum Mechanics, Gorge Allen and Unwin, L.td, 1976. 